\title{
PROPERTIES OF GROUPS OF SOLAR S-BURSTS IN THE DECAMETER BAND
}

\author{
V. Dorovskyy*, V. Melnik*, A. Konovalenko*, A. Brazhenko ${ }^{\dagger}$, \\ S. Poedts ${ }^{\ddagger}$, H. O. Rucker $\$$, and M. Panchenko $\mathbb{}$
}

\begin{abstract}
On 9 July 2013 from 5:30 UT till 13:28 UT more than 1000 S-bursts were recorded by the Ukrainian radio telescope UTR-2 operated in the frequency band 9-32 MHz. All S-bursts were low intensity events with an average flux of about 10 s.f.u. and a minimum flux as low as 0.2 s.f.u. which made their detection with small instruments practically impossible. New methods of observations allowed to retrieve the weakest S-bursts with fluxes comparable to the background level. The durations and frequency drift rates of these bursts as well as the dependencies of these parameters on frequency were found. The obtained results complement the analysis by Morosan et al. [2015] with data at lowest frequencies accessible for ground-based observations.
\end{abstract}

\section{Introduction}

S-bursts are among the shortest ones reported in the metric and decametric ranges. Their durations are considerably less than $1 \mathrm{~s}$ with the shortest reported value reaching $20 \mathrm{~ms}$ [McConnell, 1982]. The durations of other well-known short bursts, solar spikes, vary from 0.1 to $1 \mathrm{~s}$ at the mentioned frequencies [Shevchuk et al., 2016]. In addition Magdalenic et al. [2006] reported about the super-short structures of metric type IV bursts whose durations were as small as $4 \mathrm{~ms}$. S-bursts are observed exclusively at meter and decameter wavelengths [Dorovskyy et al., 2006; Melnik et al., 2010; Morosan et al., 2015]. Due to short durations and low intensities S-bursts can only be investigated by large ground-based radio telescopes equipped with high resolution and sensitive back-ends, such as LOFAR, NenuFAR, LWA, UTR-2, etc. The most recent study of the S-bursts parameters was performed by Morosan et al. [2015]. The authors have analyzed about 3000 individual

\footnotetext{
* Institute of Radio Astronomy of the National Academy of Sciences of Ukraine, Kharkov, Ukraine

${ }^{\dagger}$ Poltava Gravimetric Observatory of the National Academy of Sciences of Ukraine, Poltava, Ukraine

$\ddagger$ Katholieke Universiteit Leuven, Leuven, Belgium

$\S$ Commission for Astronomy, Austrian Academy of Sciences, Graz, Austria

$\mathbb{I}$ Space Research Institute, Austrian Academy of Sciences, Graz, Austria
} 
S-bursts observed on 9 July 2013 by the LBA antennas of LOFAR operating in the tiedarray mode in the frequency band $10-90 \mathrm{MHz}$. They have also obtained a tied-array image of the radio source associated with the group of S-bursts.

On that particular day the UTR-2 [Braude et al., 1978] and URAN-2 [Brazhenko et al., $2005]$ radio telescopes were also on duty. So there is a possibility to supplement the above mentioned investigations with data obtained by other instruments at lower frequencies, namely from 9 to $32 \mathrm{MHz}$.

\section{Observations}

On 9 July 2013 from 5:30 UT till 13:28 UT a storm of S-bursts was observed by the UTR-2 radio telescope. All S-bursts appeared to be faint events with an average flux of about 10 s.f.u. and a minimum flux reaching 0.2 s.f.u., which made their detection by small instruments practically impossible. Using a new antenna configuration as well as implementation of special processing methods allowed to distinguish low intensity S-bursts against the background emission level.

Since 2010 the observations of solar radio emission with the UTR-2 radio telescope have been carried out in the interferometric mode. In this mode separate parts of the UTR-2 antenna array were used as points of a local short-baseline interferometer. The broadband correlator for this interferometer was realized on the base of the digital spectropolarimeter DSPZ [Ryabov et al., 2010; Zakharenko et al., 2016]. The configuration shown in Figure 1 has been chosen as a standard for observations of sporadic solar radio emission.

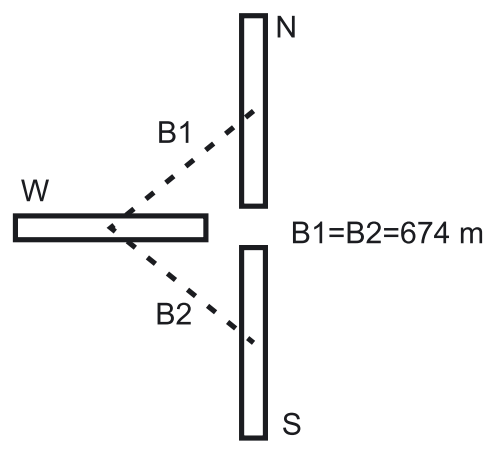

Figure 1: Local short-baseline interferometer composed of parts of the UTR-2 radio telescope.

In this scheme the geometrical centers of northern, southern and western branches of the UTR-2 radio telescope were used as the interferometer points. Thus we get two $674 \mathrm{~m}$ long mutually orthogonal bases B1 and B2 inclined to the meridian by $45^{\circ}$. Initially this configuration was implemented for finding the sizes and angular locations of the sources of solar radio bursts. In addition, it also allowed to distinguish weak small-sized sources against the background of emission from more extended sources, for instance, the quiet Sun radio emission and the galactic background. The amplitude of the correlation function at the output of an interferometer is proportional to the normalized visibility function [Thompson et al., 1986]. For the sources with Gaussian brightness distribution the normalized visibility function equals [Shepelev, 2015] 


$$
\gamma=\exp \left[-\left(\frac{\pi \theta L}{2 \sqrt{\ln 2} \lambda}\right)^{2}\right]
$$

where $\theta$ is the angular size of the source, $\lambda$ is the wavelength of the source emission, and $L$ is the length of the interferometer base. So the larger the source the smaller is the visibility function and vice versa. Taking into account the results of tied-array imaging given in Morosan et al. [2015] and the known size of the quiet radio Sun at the decameter band [Stanislavsky et al., 2013] we may expect that the sources of S-bursts are less than the size of the quiet radio Sun at the same frequency. If so then we are able to identify many weak S-bursts hidden by the background emission, as shown in Figure 2.

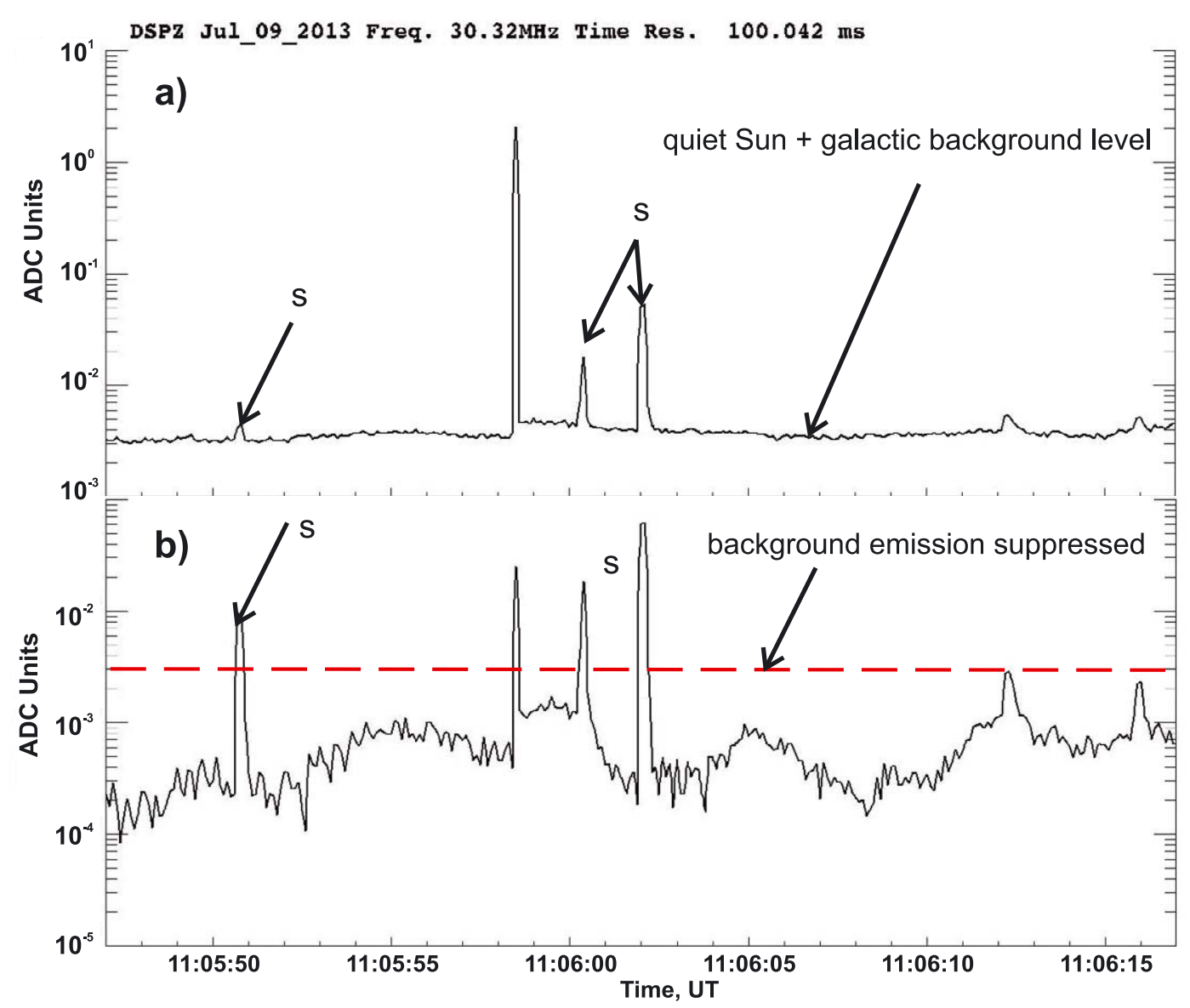

Figure 2: a) Time profile of the emission received by the northern antenna alone and b) the profile of the correlator output (base B1). The S-bursts are marked with 's'.

Using this technique more than 1000 individual S-bursts were identified in the frequency range from 9 to $32 \mathrm{MHz}$, where only some tens of S-bursts were registered by the LOFAR station [Morosan et al., 2015]. The essential feature of the observed S-bursts is that they often appear in dense groups of 5-10 individual bursts separated in time by approximately the doubled FWHM (Full Width Half Maximum) duration of one burst. Usually at these frequencies individual S-bursts extend over a rather wide frequency band, in vast majority 
exceeding $5 \mathrm{MHz}$ [Dorovskyy et al., 2005]. That was not the case for the S-bursts on 9 July 2013. Most of the S-bursts did not have a bandwidth larger than $2 \mathrm{MHz}$ (Figure 3).

The storm of S-bursts on that day was accompanied by an enhanced level of Langmuir turbulence, which manifested itself in the form of numerous type III bursts, striae and spikes, all being of moderate fluxes (see for instance Figures 3 and 4 ). The same behavior was also found by Melnik et al. [2010] and Morosan et al. [2015]. This fact may indicate that the background Langmuir turbulence associated with type III bursts most likely takes part in the processes of S-bursts generation.

An interesting phenomenon was observed when successive S-bursts of one group formed a kind of chain drifting in frequency. During the UTR-2 observations on 9 July 2013 more than 20 of such chains were identified. These chains had exclusively positive frequency drift rates varying from 1 to $5 \mathrm{MHz} \cdot \mathrm{s}^{-1}$ and consisted of 4 to 16 elements. The frequency widths of the chains varied from 1.5 to $6 \mathrm{MHz}$. Some chains are marked with yellow arrows in Figure 3. The existence of such chains was never reported before.

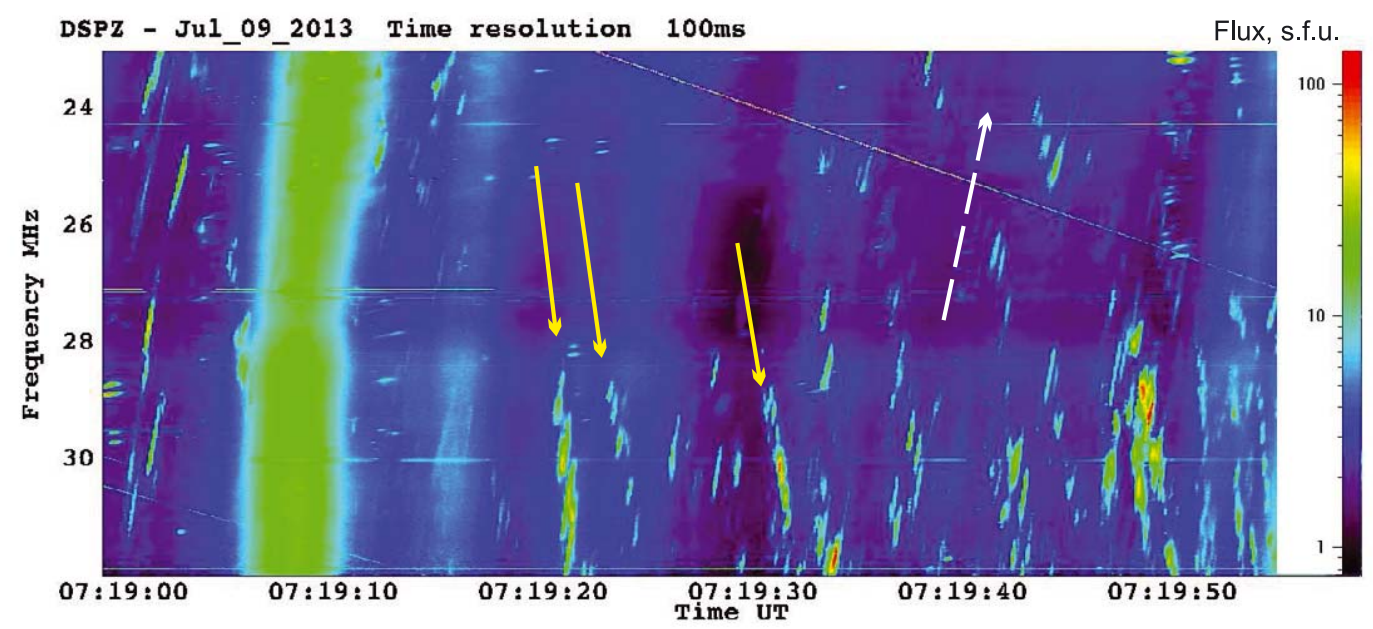

Figure 3: Dynamic spectrum of the positively drifting chains of S-bursts (some marked with yellow arrows). The white dashed arrow shows the average frequency drift of the S-bursts. The fluxes are given in s.f.u., see color bar at the right side of the figure.

Since the observations were also performed by the URAN-2 radio telescope, there was the possibility to measure the degree and the sense of circular polarization in the most powerful S-bursts. The dynamic spectrum and the spectrum showing the degree of circular polarization of radio bursts observed by the URAN-2 radio telescope are given in Figure 4. We have found that the discussed S-bursts are strongly polarized. The degree of circular polarization in average equaled $50 \%$, sometimes reaching as high as $90 \%$. The sense of polarization was always positive and opposite to that of accompanying striae and spikes.

\section{Data analysis and discussion}

The data obtained from the DSPZ spectropolarimeter was processed via dedicated IDLbased software. Because of the very low intensity the identification of the individual 


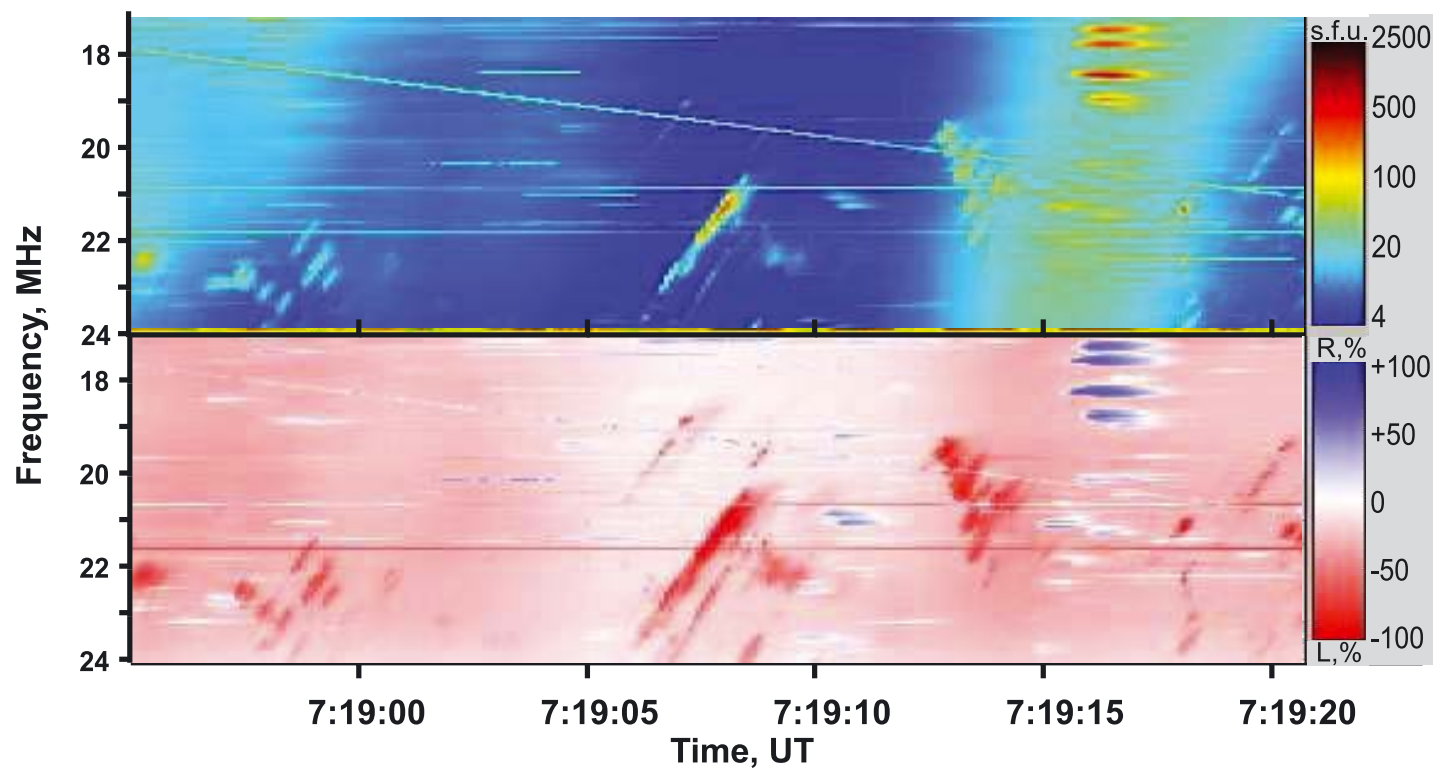

Figure 4: Power dynamic spectrum (upper panel) and polarization dynamic spectrum (bottom panel) of the S-bursts.

S-bursts was performed manually via their appearance in the time differential dynamic spectra, while their parameters were measured in semi-automatic mode using time and frequency profiles. All 1000 identified S-bursts are non-uniformly distributed across the observed frequency band. There is a strong tendency of the S-bursts occurrence rate to decrease towards low frequencies. A similar tendency was found also by the LOFAR station [Morosan et al., 2015]. These two distributions can easily be compared in Figure 5 . Note that Figure 5a shows two separate distributions for start and end frequencies of the S-bursts, while Figure 5b presents the distribution for median frequencies of these bursts.

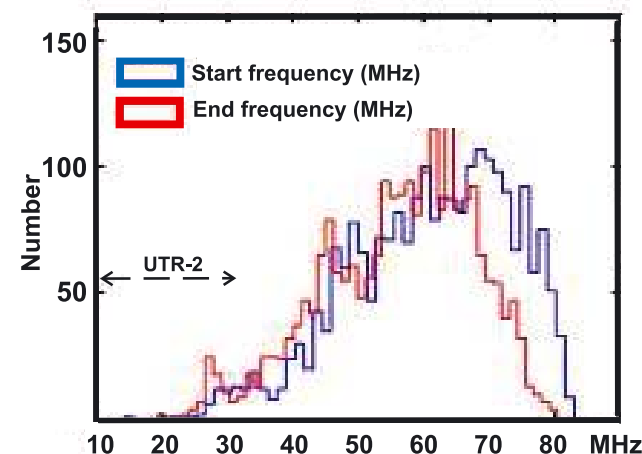

a)

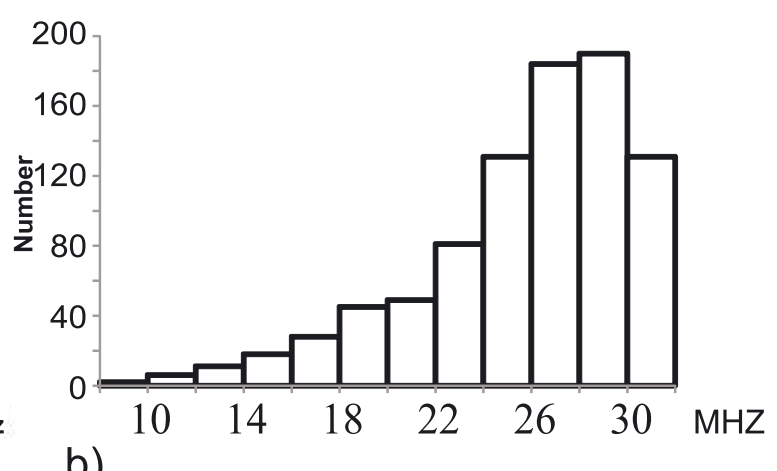

b)

Figure 5: a) S-bursts distributions by start and end frequencies observed on 9 July 2013 by the LOFAR station [Morosan et al., 2015] and b) S-bursts distribution by their median frequencies observed by the UTR-2 radio telescope.

From Figure 5 it is clearly seen that the UTR-2 radio telescope has identified about 1000 S-bursts at the frequency band where the LOFAR station has observed roughly 20 times less bursts. In our opinion this happened due to an artificial selection effect caused 
by the difference in sensitivity between the antennas of the two giant radio telescopes at frequencies below $30 \mathrm{MHz}$. In other words, most of the weakest low-frequency Sbursts were not identified by the LOFAR station because their fluxes were close to the background emission flux and comparable to the LOFAR LBA antenna noise level at these low frequencies. At the same time the decreasing number of the S-bursts observed by the UTR-2 radio telescope at frequencies above $30 \mathrm{MHz}$ (Figure 5b) has the same nature and caused by substantial drop of sensitivity at these frequencies. It also speaks in favor of good perspectives for joint, coordinated observations (LOFAR+UTR-2+URAN-2) in order to achieve excellent sensitivity in the frequency band above the ionospheric cutoff up to $240 \mathrm{MHz}$. The part distribution (Figure 5b) confined within frequencies $10-30 \mathrm{MHz}$ has been approximated by the power-law function

$$
N \approx 110 \cdot f_{25}^{3}
$$

where $N$ is the number of S-bursts counted in each frequency bin of $2 \mathrm{MHz}$, and $f_{25}$ is the central frequency of the bins normalized to $25 \mathrm{MHz}$.

Two main parameters, which distinguish S-bursts from other types of solar radio emissions, are the frequency drift rate and the duration and their dependencies on frequency. Statistical analysis shows that the average drift rate of the S-bursts observed on 9 July 2013 by the UTR-2 radio telescope is $-1.57 \pm 0.2 \mathrm{MHz} \mathrm{s}^{-1}$ and the average duration is $0.5 \pm 0.05 \mathrm{~s}$. According to McConnell [1982] drift rates of the meter band S-bursts are proportional to the frequency to the power of 1.6 .

The large amount of S-bursts analyzed in the present study enabled the determination of the dependencies of the above mentioned parameters on frequency with high reliability. The dependence of the S-bursts drift rate on frequency is shown in Figure 6 (black solid curve).

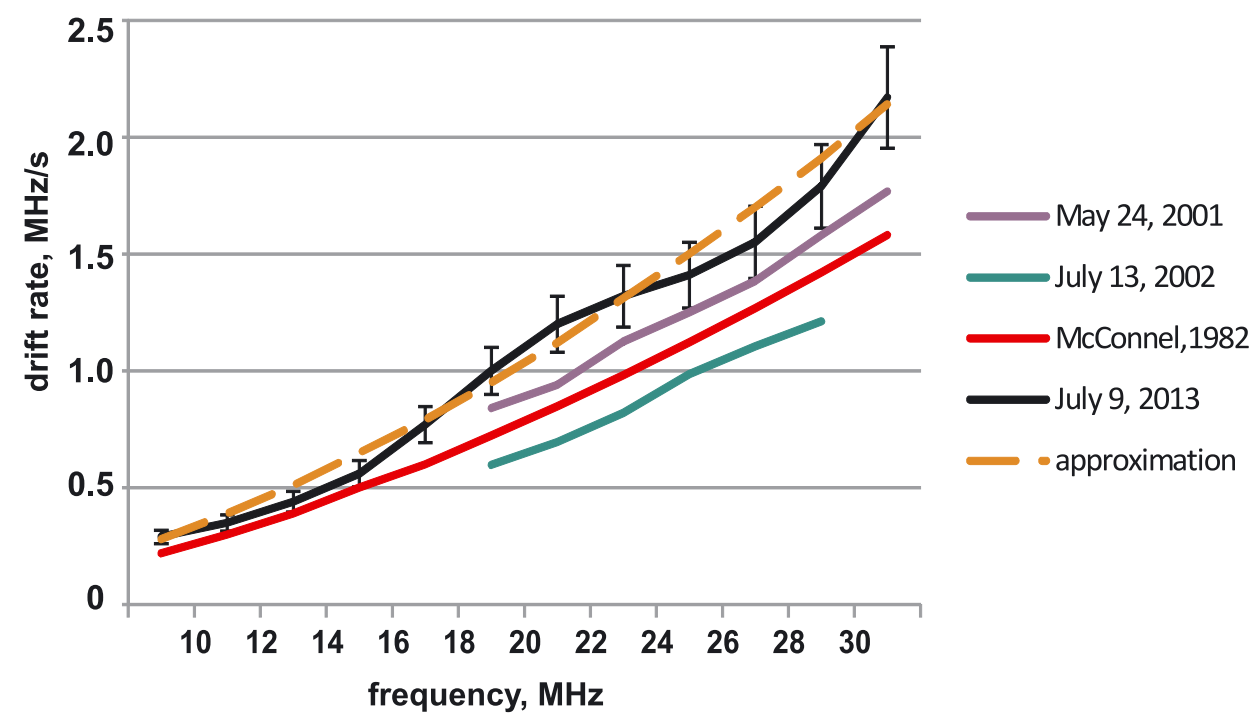

Figure 6: S-bursts drift rates dependencies on frequency obtained from different observations. 
This dependence was approximated by the power-law equation in the form of

$$
\dot{f}(f)=7.4 \cdot 10^{-3} \cdot f^{1.65}
$$

where $\dot{f}$ is the frequency drift rate in $\mathrm{MHz} \mathrm{s}^{-1}$ and $f$ is the frequency in MHz. This approximation, shown by the brown dashed curve in Figure 6, shows a similar trend like the results obtained by McConnell (solid red curve in Figure 6).

For comparison all the dependencies were plotted in the same graph together with curves for 2001 and 2002 storms of S-bursts, reported by Dorovskyy et al. [2006]. One can see that for over more than 10 years these dependencies did not change considerably. The observed differences are possibly connected with slight variations of the exciter's speed and plasma density. The wavy shape of the experimental dependence may appear due to some density inhomogeneity in the way of the exciters or by the errors of the measurements due to extremely low intensities of the bursts. The latter fact will be checked in the next experiments.

It was found by Melnik et al. [2010] that the durations of S-bursts, measured in the frequency band 20-31 MHz, did not depend on observing frequency. Apparently such conclusion was made due to the narrow frequency range of observations considered in that study. The present analysis, performed in a frequency band twice as wide, revealed such a dependence (see Figure 7).

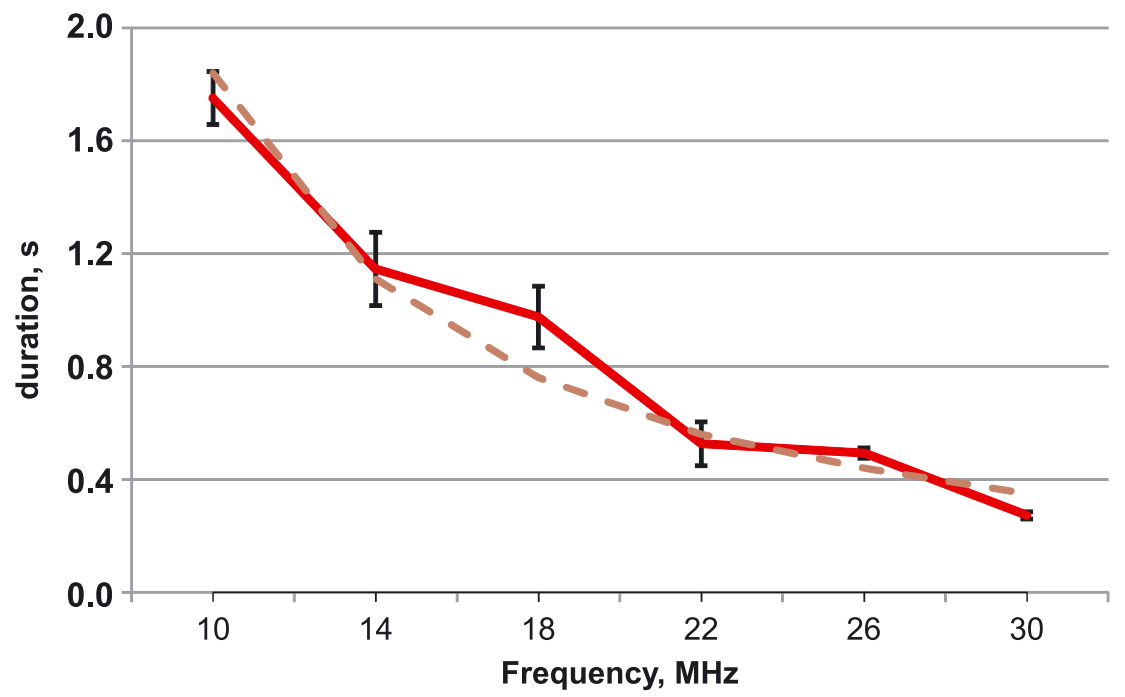

Figure 7: The dependence of the S-bursts durations on frequency obtained from observations on 9 July 2013.

The analytical expression of this dependence is shown below.

$$
\tau(f)=0.46 \cdot f_{25}^{-1.5}
$$

where $\tau$ is the duration in ms. 
The duration change with frequency may carry an important information about the plasma parameters in the emission source. Recently it was shown by Shevchuk et al. [2016] that the durations of solar spikes are possibly defined by the collision times in plasma and hence can be used as a plasma temperature diagnostic tool. The durations of S-bursts are several times shorter than the collision times estimated at the corresponding heights. So to explain such short durations some alternative interpretation is needed.

In the decameter band all known types of solar bursts, including S-bursts, are believed to occur due to plasma emission mechanism. We suppose that S-bursts are generated in the process of coalescence of Langmuir waves $l$ with fast magnetosonic waves $w$, i.e. $l+w=t$ [Melnik et al., 2010]. In this model fast magnetosonic waves $w$ are excited by the electron beams with preferred velocity equal to both phase and group velocities of these waves. Our observations indeed show that the linear velocities of the S-bursts sources, derived from the frequency drift rates, equal $\sim 0.1 c$, which corresponds to $\sim 6 v_{T_{e}}\left(v_{T_{e}}\right.$ is the electron thermal velocity) under a coronal plasma temperature of about $2 \cdot 10^{6} \mathrm{~K}$ as it was predicted by the model proposed by Melnik et al. [2010].

S-bursts are always accompanied by type III bursts, indicating a high level of the background Langmuir turbulence in the region of generation. The fact that sometimes S-bursts form positively drifting chains may be explained if we suppose that there exist waves of density and/or magnetic field which propagate towards the Sun and cross the S-bursts source, thus increasing the effectiveness of the process $l+w=t$. According to our current observations the velocities of these waves are also on average $\sim 0.1 c$. The possible candidates for that could be for instance Langmuir waves excited by low velocity beams based on spatially localized heating of plasma causing perturbations in the electron distribution function [Briand et al., 2007]. According to their simulations these electron clouds can propagate, following a ballistic trajectory, both towards and outwards the Sun at velocities of a few electron thermal velocities and generate Langmuir waves. Other possible scenarios will be discussed in a separate paper.

Our observations made with the URAN-2 radio telescope show a high degree of circular polarization of the S-bursts. It can easily be explained in the framework of the model by Melnik et al. [2010], because a transverse wave generated in the process $l+w=t$ can be predominantly an O-mode wave. Detailed analysis of this property will be done later in a forthcoming paper.

\section{Conclusions}

About 1000 S-bursts were identified in the frequency band 9-32 MHz during the observations on 9 June 2013 performed with the UTR-2 radio telescope. The large number of studied S-bursts allowed us to obtain the dependency of their drift rate and duration on the observing frequency with high accuracy. In general all properties of the studied S-bursts were in good agreement with earlier reported ones [McConnell, 1982; Melnik et al., 2010; Morosan et al., 2015]. Their drift rates are lower than those of type III bursts at the same frequency [Reid and Ratcliffe, 2014]. For the first time the dependence of the Sbursts durations on frequency was found showing the increase of the burst durations with 
the decrease of the observing frequency. Observations with the URAN-2 radio telescope allowed to measure the polarization of the most powerful S-bursts. The degree of polarization was on average about $50 \%$, and the sense was always left-handed (negative). The observed positively drifting chains of the individual S-bursts were identified for the first time. They are possibly the manifestation of two exciters moving in opposite direction in the corona. A new method of observation at the UTR-2 radio telescope has shown its efficiency in detecting of the weakest solar radio bursts against the background radio emission. The obtained results provide good perspectives for future joint multi-instrument observations involving LOFAR, NenuFAR, LWA and Ukrainian radio telescopes.

Acknowledgments. This work was partially fulfilled in the framework of the FP7 project SOLSPANET (FP7-PEOPLE-2010-IRSES-269299). The Editors thank two anonymous reviewers for their help in evaluating this paper.

\section{References}

Braude, S. Ia., A. V. Megn, B. P. Ryabov, N. K. Sharykin, and I. N. Zhuk, Decametric survey of discrete sources in the northern sky. I - The UTR-2 radio telescope: Experimental techniques and data processing, Astrophys. Space Sci., 54, 3-36, 1978.

Brazhenko, A. I., V. G. Bulatsen, R. V. Vashchishin, A. V. Frantsuzenko, A. A. Konovalenko, I.S. Falkovich, E. P. Abranin, O. M. Ulyanov, V. V. Zakharenko, A. Lecacheux, and H. O. Rucker, New decameter radiopolarimeter URAN-2, Kinematics and Physics of Celestial Bodies. Suppl. Ser., 21, 43-46, 2005.

Briand, C., A. Zaslavsky, A. Lecacheux, P. Zarka, M. Maksimovic, and A. Mangeney, The faint drifting decameter radio bursts from the solar corona, in Proc. of the Second Solar Orbiter Workshop, edited by E. Marsch, K. Tsinganos, R. Marsden, and L. Conroy, ESA-SP 641, European Space Agency, Noordwijk, Netherlands, id.56, 2007.

Dorovskyy, V.V., V.N. Melnik, A.A. Konovalenko, H. O. Rucker, E. P. Abranin, and A. Lecacheux, Observations of solar S-bursts at the decameter wavelengths, in Planetary Radio Emissions VI, edited by H. O. Rucker, W. S. Kurth, and G. Mann, Austrian Academy of Sciences Press, Vienna, 383-390, 2006.

Magdalenic, J., B. Vrsnak, P. Zlobec, A. Hillaris, and M. Messerotti, Classification and properties of supershort solar radio bursts, Astrophys. J., 642, L77-L80, 2006.

McConnell, D., Spectral characteristics of solar S bursts, Sol. Phys., 78, 253-269, 1982.

Melnik, V. N., A. A. Konovalenko, H. O. Rucker, V. V. Dorovskyy, E. P. Abranin, A. Lecacheux, and A. S. Lonskaya, Solar S-bursts at frequencies of 10-30 MHz, Solar Phys., 264, 103-117, 2010.

Morosan, D. E., et al. (52 co-authors), LOFAR tied-array imaging and spectroscopy of solar S bursts, Astron. Astrophys., 580, id.A65, 6 pp., 2015. 
Reid, H., and H. Ratcliffe, A review of solar type III radio bursts, Res. in Astron. Astrophys., 14, 773-804, 2014.

Ryabov, V. B., D. M. Vavriv, P. Zarka, B. P. Ryabov, R. Kozhin, V. V. Vinogradov, and L. Denis, A low-noise, high dynamic range digital receiver for radio astronomy applications: An efficient solution for observing radio-bursts from Jupiter, the Sun, pulsars and other astrophysical plasmas below $30 \mathrm{MHz}$, Astron. Astrophys., 510, A16, 2010.

Thompson, A. R., J. M. Moran, and G. W. Swenson, Interferometry and Synthesis in Radio Astronomy, Wiley-Interscience, New York, USA, 1986.

Shepelev, V. A., Determination of angular parameters of the sources of solar radio emission at decameter wavelengths (in Russian), Radiophys. Radioastron., 20, 20-29, 2015.

Shevchuk, N. V., V. N. Melnik, S. Poedts, V. V. Dorovskyy, J. Magdalenic, A. A. Konovalenko, A. I. Brazhenko, C. Briand, A. V. Frantsuzenko, H. O. Rucker, and P. Zarka, The storm of decameter spikes during the event of 14 June 2012, Solar Phys., 291, 211-228, 2016.

Stanislavsky, A. A., A. A. Koval, and A. A. Konovalenko, Low-frequency heliographic observations of the quiet Sun corona, Astronom. Nachrichten, 334, p.1086, 2013.

Zakharenko V.V., et al. (51 co-authors), Digital Receivers for low-frequency radio telescopes UTR-2, URAN, GURT, J. Astron. Instrumentation, 5, 4, id.1641010-738, 2016. 